\title{
RELAJACIÓN RESIDUAL EN LA UNIDAD DE CUIDADO POSANESTÉSICO
}

Luis Eduardo Reyes MD*, Ana Ruth Valencia MD**, Carlos Andrés Campo MD**, Luis Alfonso Muñoz MD***

\section{Resumen}

El uso de relajantes neuromusculares en la práctica clínica es cada día más frecuente e indispensable en el acto anestésico y la unidad de cuidado intensivo. Aunque los bloqueantes neuromusculares tanto aminoesteroideos como benzilisoquinolinas de última generación tienen efectos adversos mínimos, la relajación residual es una complicación de incidencia considerable asociada con un incremento en la morbilidad y mortalidad. Debido a esto planteamos la importancia de conocer los diferentes métodos de monitoria del bloqueo neuromuscular con su interpretación, haciendo énfasis en la monitoria con el tren de cuatro (TOF), la razón entre la primera y la cuarta respuesta T4/T1 (TOF RATIO) y estrategias de prevención de la relajación residual.

Palabras clave: monitoreo del bloqueo neuromuscular, bloqueo neuromuscular posoperatorio, drogas bloqueadoras neuromusculares de acción intermedia, unidad de cuidados posanestésicos (UCPA), parálisis residual posoperatoria (PORC).

Abreviaturas: TOF, tren de cuatro; PORC, parálisis residual posoperatoria; RNMND, relajantes neuromusculares no despolarizantes; UCPA, unidad de cuidado posoperatorio.

\section{RESIDUAL NEUROMUSCULAR BLOCKADE IN THE POST- ANESTHESIA CARE UNIT}

\section{Abstract}

The use of neuromuscular blocking agents in clinical practice is more common every day and is indispensable in anesthesia and in the intensive care unit. Although neuromuscular blocking agents, both the latest generation of aminosteroids as well as benzylisoquinolines, have minimal adverse effects, a significant incidence of residual neuromuscular blockade constitutes a complication associated with increased morbidity and mortality. For this reason we advocate the importance of knowledge on the various neuromuscular block monitoring methods and their interpretation, emphasizing train-of-four (TOF) stimulation, ratio between the first response to the fourth response T4/T1 (TOF RATIO) and prevention strategies of residual neuromuscular blockade.

Key words: Monitoring neuromuscular block, postoperative neuromuscular blockade, intermediate acting neuromuscular blocking agents, post-anesthesia care unit (PACU), postoperative residual paralysis (PORP)

Fecha recibido: junio 30 de 2015 - Fecha aceptado: agosto 13 de 2015

* Jefe de programa de posgrado en Anestesiología y Reanimación, Hospital de San José. Profesor Asistente, Fundación Universitaria de Ciencias de la Salud. Bogotá DC, Colombia.
** Residente III de Anestesiología y Reanimación, Fundación Universitaria de Ciencias de la Salud. Bogotá DC, Colombia.

*** Instructor Asociado Fundación Universitaria de Ciencias de la Salud. Anestesiólogía y Reanimación. Hospital de San José. Bogotá DC, Colombia. 


\section{Introducción}

Cerca de las dos terceras partes de los procedimientos anestésicos a nivel mundial se realizan bajo anestesia general. ${ }^{1}$ De estos el $60 \%$ reciben algún tipo de relajante neuromuscular, ya sea despolarizante o no. La implementación rutinaria de los relajantes musculares no despolarizantes (RNMND) de acción intermedia, ha demostrado una reducción significativa de la parálisis residual posoperatoria PORC (por sus siglas en ingles) en la unidad de cuidados posanestésicos (UCPA). ${ }^{2,3}$ A pesar de esto, se estima que de 15 a $88 \%$ de los pacientes que ingresan a esta unidad tienen algún efecto de bloqueo neuromuscular, desarrollando complicaciones posoperatorias respiratorias como obstrucción de la vía aérea superior e hipoxemia con una incidencia entre $1.3 \%$ y $6.9 \% 1,{ }^{2-4}$ además del riesgo de broncoaspiración, retraso en tiempos de recuperación y de egreso de la UCPA. ${ }^{5,6}$ La incidencia de la relajación neuromuscular es variable, distintos estudios muestran un rango de $5 \%$ a $85 \%$, que se puede explicar por las diferentes metodologías, mediciones y manejos perioperatorios. ${ }^{1}$

\section{Parálisis residual posoperatoria}

Desde 1970 con la introducción del tren de cuatro (TOF) (por sus siglas en ingles) y la correlación de la relación TOFR (relación de la amplitud de la primera respuesta con la cuarta) con signos clínicos, Ali y col..$^{7-9}$ demostraron en seis voluntarios sanos despiertos una correlación entre capacidad vital, fuerza inspiratoria y espiratoria con un $\mathrm{TOFR} \geq 0.7$. Desde entonces y durante dos décadas se consideró este el umbral para excluir la parálisis residual posoperatoria. En la actualidad el consenso general para excluir parálisis residual clínica se logra con una relación TOF $>0.9$, medida mediante mecanomiografía o electromiografía, o un valor de $1.0 \mathrm{si}$ para su medición se utiliza aceleromiografía. ${ }^{8,9}$ Por lo anterior, la relajación residual se define como un $\mathrm{TOF}<0.9$ asociado con la presencia de signos y síntomas de debilidad muscular durante el periodo posoperatorio. ${ }^{10,11}$

\section{Estrategias de prevención de la relajación residual}

Hay tres estrategias para evitar la parálisis residual y sus consecuencias. La primera, de elección por anestesiólogos angloamericanos es la reversión farmacológica de todos los RMND con inhibidores de colinesterasa, opuesta a la tendencia europea de reversión farmacológica, solo se acerca al $25 \%$ de los casos que es semejante a lo nuestro. ${ }^{12,13}$ La segunda es evitar la sobredosificación de RMND con monitoria estricta neuromuscular. ${ }^{14}$ La tercera consiste en evitar el uso de RMNDP en ausencia de indicación quirúrgica y donde exista la posibilidad de asegurar la vía aérea con dispositivos supraglóticos. ${ }^{15}$

\section{Relajantes musculares no despolarizantes de acción intermedia y relajación residual posoperatoria}

Clásicamente los (RMND) se han clasificado en dos grupos basados en sus características químicas, lo que a su vez se asocia con distintos efectos adversos. El grupo de los aminoesteroideos se relacionan con efectos vagolíticos y el de las benzilisoquinolinas con liberación de histamina. Los RMND de última generación de ambos grupos no presentan efectos adversos de importancia clínica relevante, a pesar de tener características estructurales similares. ${ }^{16}$ Las últimas poseen una recuperación rápida, predecible y espontánea de la relajación comparada con los primeros. Los estudios publicados a partir de 2000 en los cuales se utilizaron RMND de acción intermedia muestran persistencia de TOFR $<0.9$ en las UCPA, de causa multifactorial como son las dosis más altas para intubación (2 a 4 veces, DE 95\%) para acortar el inicio de acción, la variabilidad individual del tiempo de recuperación y la administración de RMND y las infusiones de RMND. ${ }^{4}$

\section{Valoración de la parálisis residual}

El uso de monitoria cuantitativa neuromuscular permite optimizar el manejo de los RMND y por tanto 
en los fármacos para su reversión, pero en la práctica clínica el panorama es diferente. En 2007 Grayling y col. un estudio de doce servicios de anestesia en el Reino Unido demostró que solo el 10\% de los anestesiólogos utiliza de manera rutinaria monitoría de la relajación y más del $60 \%$ nunca usa un monitor. ${ }^{17,18}$ La monitoria cualitativa de uso más frecuente presenta un punto ciego entre TOFR de 0.5 a 0,95 para determinar relajación residual, ya que solo de 37 a $57 \%$ de los anestesiólogos detectan visualmente un desvanecimiento de la respuesta motora con un TOFR >0.4.4.

\section{Pruebas clínicas para bloqueo residual}

Se han descrito múltiples para excluir el diagnóstico de PORC, pero no son específicas para predecir la función respiratoria ni se deben usar para inferir la adecuada función muscular respiratoria ${ }^{2,13,19}$ (Tabla 1).

\section{Monitoría subjetiva}

La monitoria subjetiva o cualitativa consiste en la evaluación táctil o visual de la respuesta a la estimulación de un nervio periférico. Es el método más común de medición en salas de cirugía UCPA y UCI, con una utilidad limitada ya que solo proporciona información binaria acerca de la presencia o no de fatiga muscular (Tabla 2). ${ }^{16} \mathrm{El}$ uso de patrón de doble ráfaga proporciona una mejor determinación subjetiva cuando se compara con el TOF.

\begin{tabular}{|l|c|c|c|}
\hline \multicolumn{3}{|c|}{ Tabla I. Pruebas clínicas para excluir el } \\
diagnóstico de PORC \\
\hline \multicolumn{1}{|c|}{ Prueba } & $\begin{array}{c}\text { Correlación } \\
\text { TOFR }\end{array}$ & Sensibilidad & VPP* \\
\hline Sostén cefálico & $0.45-0.75$ & $87.7 \%$ & \\
\hline $\begin{array}{l}\text { Protrusión de la } \\
\text { lengua }\end{array}$ & $<0.86(13)$ & $90 \%$ & 0.51 \\
\hline $\begin{array}{l}\text { Prensión de la mano } \\
\text { durante 5 segundos }\end{array}$ & $<0.5$ & $89 \%$ & 0.51 \\
\hline
\end{tabular}

*VPP:Valor predictivo positivo

\section{Monitoría objetiva, aceleromiografía}

Introducida en 1988, es la más utilizada hoy en la práctica clínica, debido a su relativo bajo costo y fácil uso, comparada con la electromiografía. ${ }^{19}$ Después de la colocación adecuada de los electrodos de estimulación (previa limpieza de piel con alcohol $<6 \mathrm{~cm}$, electrodo negativo en posición distal y temperatura de la piel no $<32$ grados centígrados), se localiza una pieza eléctrica sobre el músculo correspondiente al nervio objetivo. El movimiento de este músculo genera un voltaje sobre la pieza eléctrica que se correlaciona con la aceleración del músculo. ${ }^{19,20}$ En 2008 se realizó un metaanálisis en el cual se incluyeron todos los artículos relacionados con el uso de aceleromiografía hasta $2007^{20}$, encontrando las siguientes directrices basadas en la evidencia Grado A: la aceleromiografía es más sensible en el diagnóstico de PORC que las pruebas clínicas.

Grado B: la aceleromiografía es más sensible que la valoración subjetiva visual o táctil, tanto para el TOF como para la doble ráfaga y la estimulación tetánica.

Grado C: la monitoria perioperatoria con aceleromiografía proporciona detección de PORC y es tan útil como la mecanomiografía para el diagnóstico de esta.

No hay evidencia suficiente para decidir si TOFR no corregido o no normalizado (dividir el valor final del TOF de recuperación entre el valor de control determinado antes de la administración del RMND) debe ser 0.9 o 1.0 o mayor para excluir el diagnóstico de PORC. ${ }^{21}$

\section{Mecanomiografía}

Es el patrón de oro para la monitoría neuromuscular. Mide la fuerza de contracción isométrica del músculo a una estimulación nerviosa. El sitio más frecuente es a nivel del nervio cubital con estimulación del aductor del pulgar con una precarga de 200 o 300 g e inmovilización del brazo para la estabilización de la señal (acelerómetro más precarga = mecanógrafo). $\mathrm{Su}$ uso en la práctica clínica diaria se encuentra limitado por el tiempo que toma la preparación preprueba y lo voluminoso del equipo. ${ }^{19,20}$ 


\begin{tabular}{|c|c|c|c|c|c|c|}
\hline $\begin{array}{l}\text { Patrón de } \\
\text { estimulación }\end{array}$ & Realización & $\begin{array}{c}\text { Correlación TOFR y } \\
\text { BNM }\end{array}$ & $\mathbf{S}$ & $\mathbf{E}$ & VPP* & \\
\hline $\begin{array}{l}\text { Tren de cuatro } \\
\text { (TOF) }\end{array}$ & $\begin{array}{l}\text { Cuatro descargas de } 2 \mathrm{~Hz} \text { cada } \\
0.5 \text { segundos con un intervalo mí- } \\
\text { nimo entre cada estímulo de } 10 \text { se- } \\
\text { gundos }\end{array}$ & $\begin{array}{l}\text { T4 }=\text { BNM } 75-80 \% \\
\text { T3 }=\text { BNM } 85 \% \\
\text { T2 }=\text { BNM } 90 \% \\
\text { TI }=\text { BNMI00\% }(25)\end{array}$ & $98 \%$ & $14 \%$ & $95 \%$ & $\begin{array}{l}\text { Para procedimientos quirúr- } \\
\text { gicos se considera adecuado } \\
\text { nivel de relajación hasta que } \\
\text { reaparece la segunda res- } \\
\text { puesta }\end{array}$ \\
\hline $\begin{array}{l}\text { Estimulación } \\
\text { tetánica }\end{array}$ & $\begin{array}{l}\text { Estimulación bifásica de } 200 \text { mili- } \\
\text { segundos a una alta frecuencia ( } 50 \\
-100 \mathrm{~Hz} \text { ) durante } 5 \text { segundos lo que } \\
\text { equivale a un estímulo cada } 20 \mathrm{~ms} \\
\text { durante } 5 \text { segundos }(17,24,25)\end{array}$ & $\begin{array}{l}\text { TOFR } 0.8-0.9 \mathrm{con} \\
100 \mathrm{~Hz} \\
\text { TOFR } 0.4 \text { con } 50 \mathrm{~Hz}\end{array}$ & $74 \%$ & $55 \%$ & $85 \%$ & $\begin{array}{l}\text { Uso clínico limitado en pa- } \\
\text { cientes despiertos ya que } \\
\text { es doloroso y la facilitación } \\
\text { postetánica por un periodo } \\
\text { de } 5 \text { a } 10 \text { minutos posesti- } \\
\text { mulación altera la valoración } \\
\text { de cualquier otro estímulo }\end{array}$ \\
\hline $\begin{array}{l}\text { Cuenta } \\
\text { postetánica } \\
\text { (PTC) }\end{array}$ & $\begin{array}{l}\text { Descarga de } 50 \mathrm{~Hz} \text { durante } 5 \text { se- } \\
\text { gundos, seguido de un periodo de } \\
3 \text { segundos, de } 10 \text { o } 20 \text { estímulos } \\
\text { simples de I Hz }\end{array}$ & $\begin{array}{l}\mathrm{PTC}=2, \mathrm{TI} \text { en } 25 \circ \\
30 \text { minutos } \\
\mathrm{PTC}=6, \mathrm{TI} \text { en } 5 \circ \\
10 \text { minutos } \\
\mathrm{PTC}=\circ>7, \mathrm{TI}<5 \\
\text { minutos }\end{array}$ & & & & $\begin{array}{l}\text { Permite la evaluación visual } \\
\text { o táctil de la profundidad del } \\
\text { bloqueo con RMND que no } \\
\text { responde a estimulación con } \\
\text { TOF, estímulo simple, DBS o } \\
\text { tétano, utilizando el fenóme- } \\
\text { no de facilitación postetánica. }\end{array}$ \\
\hline Doble ráfaga & $\begin{array}{l}\text { Dos ráfagas (una consiste en } 2 \text { ○ } 3 \\
\text { impulsos) de estímulo a } 50 \mathrm{~Hz} \text { con } \\
\text { un intervalo de } 750 \mathrm{~ms}\end{array}$ & $\begin{array}{l}\text { La fatiga del segundo im- } \\
\text { pulso comparado con el } \\
\text { primero }=\text { TOFR }<0.6\end{array}$ & $99 \%$ & $14 \%$ & $100 \%$ & \\
\hline
\end{tabular}

*VPP:Valor predictivo positivo

\section{Electromiografía}

Valora la actividad eléctrica del músculo estimulado. La respuesta electromiográfica se mide con electrodos ubicados en la eminencia tenar, hipotenar o el primer músculo interóseo de la mano. Sin embargo la señal puede afectarse por numerosos factores como son los electrodos, hipotermia, interferencia eléctrica y la estimulación directa muscular. ${ }^{22,23}$

\section{Antagonismo de la relajación residual}

La controversia acerca del uso rutinario de la reversión farmacológica del bloqueo neuromuscular se soporta en el hecho de que el uso de inhibidores de la colinesterasas por si mismos implica un riesgo y un costo metabólico alto para el paciente. ${ }^{24,25}$ Los inhibidores de la acetilcolinesterasa disminuyen el metabolismo de la acetilcolina en la placa neuromuscular permitiendo un aumento en su concentración, superando así los efectos de los RMNDP. No obstante estos fármacos tienen como desventaja el mecanismo indirecto de reversión con baja efectividad y seguridad en bloqueos musculares profundos, efectos poco predecibles y su acción no selectiva sobre los receptores nicotínicos que desencadena efectos adversos cardiovasculares y respiratorios $^{26}$ (Tabla 3).

La administración de antimuscarínicos como atropina o glicopirrolato lleva a disfunción del control parasimpático del corazón con disminución en la sensibilidad de los barorreceptores y variabilidad en la frecuencia cardiaca. ${ }^{26}$ En la actualidad se encuentra en introducción de la práctica clínica como alternativa para los anticolinesterásicos el sugamadex, fármaco diseñado para encapsular los RMND esteroideos, rocuronio y vecuronio. Permite revertir cualquier grado de bloqueo neuromuscular al ajustar la dosis, reduciendo el riesgo de recurarización. La interacción fármaco-fármaco es de acción extrarápida, limitada por el tiempo de circulación sin alteración por su eliminación renal. Las indicaciones actuales para el uso de sugamadex radican en pacientes con miastenia gravis, neumopa- 


\section{Tabla 3. Efectos de anticolinesterasicos}

\begin{tabular}{|l|l|}
\hline $\begin{array}{l}\text { Efectos indirectos } \\
\text { sobre receptores } \\
\text { muscarínicos }\end{array}$ & $\begin{array}{l}\text { Salivación, bradicardia, asistolia, bronco- } \\
\text { constricción e incremento en la motilidad } \\
\text { intestinal }\end{array}$ \\
\hline $\begin{array}{l}\text { Náusea } \\
\text { posoperatoria }\end{array}$ & Dosis mayores de 50 mcg/k de neostigmina \\
\hline Otros & $\begin{array}{l}\text { Aumento crítico de la tensión en la anasto- } \\
\text { mosis en el intestino delgado, colon y recto } \\
\text { en un } 200 \%\end{array}$ \\
\hline Debilidad & $\begin{array}{l}\text { Secundario a administración de neostig- } \\
\text { mina a dosis habituales en pacientes con } \\
\text { recuperación completa de la función neu- } \\
\text { romuscular; lleva a falla en la trasmisión } \\
\text { neuromuscular, con fatiga en el conteo TOF, } \\
\text { por desensibilización de los receptores de } \\
\text { acetilcolina, bloqueo de la despolarización } \\
\text { o apertura de los canales bloqueados, alte- } \\
\text { rando de manera significativa todo los gru- } \\
\text { pos musculares. }\end{array}$ \\
\hline
\end{tabular}

tía o cardiopatía, bloqueo profundo de la relajación e intubación fallida. ${ }^{27-29}$

No es del todo cierto que eliminar la monitoria de la relajación además de aplicar una dosis única de intubación (2 DE 95) de un RMNDP de duración intermedia, conducirá a una recuperación espontánea de la función neuromuscular en los siguientes 90 minutos después de su aplicación y cumplido este tiempo la reversión farmacológica no es necesaria. Los estudios realizados por Caldwell en 1993 con 20 voluntarios sanos a los cuales se le administró una única dosis de vecuronio a $0.1 \mathrm{mg} / \mathrm{k}$ reveló que en cuanto el tiempo de recuperación del TOFR, dos horas después veinte de los sujetos presentaron un TOFR $<0.75$, dos de estos pacientes tuvieron TOFR $<0.50$ a las tres horas posadministración del medicamento y tres horas después, diez de los veinte presentaron TOFR entre 0.6 y 0.7 . Por tanto, se considera como la mejor práctica médica la monitoria objetiva cuantitativa de la relajación que documente el retorno de la función muscular, cumpliendo estándares de recuperación TOFR de 0.9. ${ }^{6,17,24,30}$

\section{Reversión con monitoría objetiva cuantitativa}

Thomas en 2010 en un estudio con 120 pacientes llevados a cirugía, utilizando atracurio como RMND y TOFR entre 0.4 y 0.6 y a quienes se administraron dosis bajas de neostigmina (20 a $30 \mathrm{mcg} / \mathrm{k}$ ), midieron los niveles de recuperación de la función muscular diez minutos después de la reversión farmacológica. En el mismo estudio resalta los cambios de valor de TOFR como criterio de recuperación de la función neuromuscular de 0.7 a 0.9 sin adaptación acorde con la dosis de neostigmina. ${ }^{25}$ Entonces la pregunta es: ¿Se debe esperar un porcentaje de recuperación mayor en la función neuromuscular antes del inicio de la reversión farmacológica? Múltiples estudios reportan de 20 a 30 minutos después de la dosis habitual de neostigmina antes de lograr TOF de 4 o TOFR 0.9. Pero se necesitan estudios concluyentes para poder determinar el grado de relajación prerreversión farmacológica. ${ }^{31}$

Hoy se considera que con TOFR $<0.9$ se debe administrar un antagonista de la relajación muscular si se planea extubar al paciente. Las dosis establecidas según el grado de bloqueo neuromuscular son: 1) TOFR $>0.9$ no reversión; 2) TOFR 0.4 a 0.9 neostigmina 15 a 25 $\mathrm{mcg} / \mathrm{k}$; 3) TOFR<0.4 neostigmina $50 \mathrm{mcg} / \mathrm{k}$; y 4) ante la no respuesta a TOF esperar al menos dos respuestas para iniciar la reversión farmacológica. ${ }^{31}$

\section{Reversión con monitoría objetiva cualitativa}

La utilización del estimulador de nervio convencional permite tomar la decisión de reversión farmacológica así: 1) ante cuatro respuestas con fatiga en forma visual o táctil, administrar neostigmina $40 \mathrm{mcg} / \mathrm{k} ; 2$ ) cuando hay cuatro respuestas sin fatiga, es importante tener en cuenta la baja sensibilidad, especificidad y valor predictivo positivo de la prueba y pensar en la posibilidad de PORC, por lo cual se debe considerar la reversión farmacológica con dosis de $15 \mathrm{mcg} / \mathrm{k} ; 3$ ) con dos a tres respuestas, usar neostigmina $50 \mathrm{mcg} / \mathrm{k}$; y 4) ante la no respuesta a TOF esperar hasta obtener cuatro respuestas de TOF antes de iniciar la reversión farmacológica con neostigmina. ${ }^{32,33}$

\section{Reversión farmacológica sin monitoría de la función muscular}

Aunque la no utilización de la monitoria de la relajación se considera una práctica clínica inapropiada 
y que las pruebas clínicas para determinar la función neuromuscular no tienen suficiente especificidad o sensibilidad para considerarse criterio de recuperación, es necesario el uso de reversión de la relajación muscular en todos los pacientes que reciben RMND de acción intermedia y dependiendo de la circunstancias disminuir a criterio clínico la dosis de neostigmina. ${ }^{33,34}$

\section{Complicaciones}

A pesar de que en el escenario clínico es difícil establecer una relación lineal entre efectos adversos respiratorios posoperatorios y los relajantes musculares, múltiples estudios clínicos recientes han podido demostrar asociación entre estos con la recuperación clínica y el riesgo para desarrollar complicaciones respiratorias críticas después de la administración de anestesia general. ${ }^{34}$

\section{Efectos en la función respiratoria}

Desde la década de los 70 se estableció una clara relación entre bajos valores de TOF $(<0.7)$ y alteración en la función respiratoria con variación en el volumen corriente, capacidad vital y fuerza inspiratoria y espiratoria. Glen en 2008 en un estudio retrospectivo que involucró 7459 pacientes posoperatorios, encontró eventos respiratorios críticos definidos por hipoxemia, alteración en la ventilación y obstrucción de la vía aérea en el $0.8 \%$, relacionado con un promedio de $\mathrm{TOF}<0.7$. El evento respiratorio crítico más frecuente fue hipoxemia en el $0.5 \%$ de los pacientes; el autor sugiere como causa probable una alteración en la respuesta ventilatoria hipóxica asociada con disfunción de los músculos faríngeos y obstrucción de la vía aérea superior. ${ }^{34} \mathrm{El}$ segundo evento respiratorio reportado en frecuencia fue obstrucción de la vía aérea superior en el $0.3 \%$ de los pacientes. La reintubación se presentó en $0.1 \%$, datos que se correlacionan con los hallazgos de Matwes en 1990 quien encontró asociación entre reintubación y TOFR $<0.5$. Además de las alteraciones en la mecánica de la ventilación, durante la parálisis neuromuscular parcial la ventilación minuto se mantiene por un incremento en la frecuencia respiratoria, con lo cual se logran niveles de normocapnia, pero con alteración significativa de la respuesta ventilatoria hipóxica. ${ }^{32}$ Varios estudios evidencian una reducción del $30 \%$ en la respuesta ventiladora hipóxica con valores de TOFR $<0.9$, secundarios según modelos animales a una disminución de la neurotransmisión en el cuerpo carotídeo debido a la inhibición de los receptores nicotínicos por los RMND. ${ }^{7}$

\section{Síntomas asociados con PORC}

La parálisis muscular residual produce síntomas poco placenteros en los pacientes, además prolongan el tiempo de estadía en la UCPA. Fueron descritos por Kopman en 1997 basados en estudios realizados en diez voluntarios ASA I a los cuales se le administró mivacurio, quienes reportaron diplopia y alteraciones visuales con TOF 0.65 a 0.75 . Valores de TOFR entre 0.7 y 0.75 se asociaron con diplopia, incapacidad para sentarse y para oponer incisivos, debilidad facial y fuerza de agarre, dificultad para tragar o hablar; sin embargo evidenciaron una gran variabilidad de la sintomatología entre los pacientes para diferentes valores de TOF. ${ }^{17}$

\section{Conclusiones}

Los relajantes musculares mejoran la calidad de la laringoscopia, facilitan la intubación y la ventilación mecánica disminuyendo la morbilidad laríngea. La prolongación en los tiempos de relajación es multifactorial dada por dosis altas para intubación, técnicas anestésicas balanceadas, variabilidad individual al tiempo de recuperación, condiciones del paciente al ingresar a la UCPA, acidosis, hipotermia e hipercapnia. La parálisis residual posoperatoria es una complicación frecuente en anestesia y se asocia con debilidad de los músculos respiratorios superiores, obstrucción de la vía aérea, atenuación de la respuesta ventilatoria hipóxica, falla respiratoria aguda y síntomas desagradables asociados con debilidad muscular. La monitoria objetiva cuantitativa de la relajación se considera la mejor práctica clínica cuando se administran RMND. 


\section{Referencias}

1. Brull SJ, Murphy GS. Residual neuromuscular block: lessons unlearned. Part II: methods to reduce the risk of residual weakness. Anesth Analg. 2010;111(1): 129-40.

2. Combes X, Andriamifidy L, Dufresne E, Suen P, Sauvat S, Scherrer E, et al Comparison of two induction regimens using or not using muscle relaxant: impact on postoperative upper airway discomfort. Br J Anaesth. 2007;99(2):276-81.

3. Andersen BN, Madsen JV, Schurizek BA, Juhl B. Residual curarisation: a comparative study of atracurium and pancuronium. Acta Anaesthesiol Scand. 1988;32(2):79-81

4. Claudius C, Garvey LH, Viby-Mogensen J. The undesirable effects of neuromuscular blocking drugs. Anaesthesia. 2009;64 Suppl 1:10-21.

5. Craig RG, Hunter JM. Neuromuscular blocking drugs and their antagonists in patients with organ disease. Anaesthesia. 2009;64 Suppl 1:55-65.

6. Murphy GS. Residual neuromuscular blockade: incidence, assessment, and relevance in the postoperative period. Minerva Anestesiol. 2006;72(3):97-109.

7. Naguib M, Kopman AF, Lien CA, Hunter JM, Lopez A, Brull SJ. A survey of current management of neuromuscular block in the United States and Europe. Anesth Analg. 2010;111(1):110-9.

8. Plaud B, Debaene B, Donati F, Marty J. Residual paralysis after emergence from anesthesia. Anesthesiology. 2010;112(4):1013-22.

9. Capron F, Alla F, Hottier C, Meistelman C, Fuchs-Buder T. Can acceleromyography detect low levels of residual paralysis? A probability approach to detect a mechanomyographic train-of-four ratio of 0.9. Anesthesiology. 2004;100(5): 1119-24.

10. Ali HH, Wilson RS, Savarese JJ, Kitz RJ. The effect of tubocurarine on indirectly elicited train-of-four muscle response and respiratory measurements in humans. Br J Anaesth. 1975;47(5):570-4.

11. Murphy GS, Szokol JW, Marymont JH, Franklin M, Avram MJ, Vender JS. Residual paralysis at the time of tracheal extubation. Anesth Analg. 2005;100(6):18405 .

12. Cammu G, De Witte J, De Veylder J, Byttebier G, Vandeput D, Foubert L, et al Postoperative residual paralysis in outpatients versus inpatients. Anesth Analg. 2006;102(2):426-9.

13. Fuchs-Buder T, Hofmockel R, Geldner G, Diefenbach C, Ulm K, Blobner M. [The use of neuromuscular monitoring in Germany]. Anaesthesist. 2003;52(6):522-6.

14. Maybauer DM, Geldner G, Blobner M, Pühringer F, Hofmockel R, Rex C, et al. Incidence and duration of residual paralysis at the end of surgery after multiple administrations of cisatracurium and rocuronium. Anaesthesia. 2007;62(1):12-7.

15. Fawcett WJ, Dash A, Francis GA, Liban JB, Cashman JN. Recovery from neuromuscular blockade: residual curarisation following atracurium or vecuronium by bolus dosing or infusions. Acta Anaesthesiol Scand. 1995;39(3):288-93.

16. Ronald MD. Miller Anestesia. Elsevier ed2011.

17. Kopman AF, Yee PS, Neuman GG. Relationship of the train-of-fourfade ratio to clinical signs and symptoms of residual paralysis in awake volunteers. Anesthesiology. 1997;86(4):765-71.
18. Grayling M, Sweeney BP. Recovery from neuromuscular blockade: a survey of practice. Anaesthesia. 2007;62(8):806-9.

19. Fuchs-Buder T, Schreiber JU, Meistelman C. Monitoring neuromuscular block: an update. Anaesthesia. 2009;64 Suppl 1:82-9.

20. Claudius C, Viby-Mogensen J. Acceleromyography for use in scientific and clinical practice: a systematic review of the evidence. Anesthesiology. 2008; 108(6):1117-40

21 Naguib M, Lien CA, Meistelman C. Anesthesia. 6 ed. Philadelphia: Elsevier/ Churchill Livingstone; 2005. Chapter 34, Pharmacology of Neuromuscular Blocking Drugs; 958-994.e8.

22. Cai YR, Xu J, Chen LH, Chi FL. Electromyographic monitoring of facial nerve under different levels of neuromuscular blockade during middle ear microsurgery. Chin Med J (Engl). 2009;122(3):311-4.

23. Jellish WS, Leonetti JP, Buoy CM, Sincacore JM, Sawicki KJ, Macken MP. Facial nerve electromyographic monitoring to predict movement in patients titrated to a standard anesthetic depth. Anesth Analg. 2009;109(2):551-8.

24. Murphy GS, Szokol JW, Marymont JH, Greenberg SB, Avram MJ, Vender JS Residual neuromuscular blockade and critical respiratory events in the postanesthesia care unit. Anesth Analg. 2008;107(1):130-7.

25. Fuchs-Buder T, Meistelman C, Alla F, Grandjean A, Wuthrich Y, Donati F. Antagonism of low degrees of atracurium-induced neuromuscular blockade: doseeffect relationship for neostigmine. Anesthesiology. 2010;112(1):34-40.

26. Caldwell JE. Clinical limitations of acetylcholinesterase antagonists. J Crit Care. 2009;24(1):21-8.

27. Bom A, Hope F, Rutherford S, Thomson K. Preclinical pharmacology of sugammadex. J Crit Care. 2009;24(1):29-35.

28. Jones RK, Caldwell JE, Brull SJ, Soto RG. Reversal of profound rocuronium-induced blockade with sugammadex: a randomized comparison with neostigmine. Anesthesiology. 2008;109(5):816-24

29. Naguib M. Sugammadex: another milestone in clinical neuromuscular pharmacology. Anesth Analg. 2007;104(3):575-81.

30. Claudius C, Karacan H, Viby-Mogensen J. Prolonged residual paralysis after a single intubating dose of rocuronium. Br J Anaesth. 2007;99(4):514-7.

31. Arain SR, Kern S, Ficke DJ, Ebert TJ. Variability of duration of action of neuromuscular-blocking drugs in elderly patients. Acta Anaesthesiol Scand. 2005;49(3):312-5

32. Herbstreit F, Peters J, Eikermann M. Impaired upper airway integrity by residual neuromuscular blockade: increased airway collapsibility and blunted genioglossus muscle activity in response to negative pharyngeal pressure. Anesthesiology. 2009;110(6):1253-60.

33. Lee C, Katz RL. Clinical implications of new neuromuscular concepts and agents: so long, neostigmine! So long, sux! J Crit Care. 2009;24(1):43-9.

34. Eikermann M, Vogt FM, Herbstreit F, Vahid-Dastgerdi M, Zenge MO, Ochterbeck $\mathrm{C}$, et al. The predisposition to inspiratory upper airway collapse during partial neuromuscular blockade. Am J Respir Crit Care Med. 2007;175(1):9-15. 